\title{
Elimination of hepatitis C: What would be the practical approach?
}

\author{
Hyung Joon Yim \\ Department of Internal Medicine, Korea University College of Medicine, Seoul, Korea
}

Keywords: Hepacivirus; Hepatitis C, Chronic; RNA; Reflex

\section{See Article on Page 136}

Chronic hepatitis C (CHC) is a major cause of liver cirrhosis and hepatocellular carcinoma. ${ }^{1}$ Frequent changes in the genetic sequences of hepatitis $\mathrm{C}$ virus (HCV) have led to failures in vaccine development, ${ }^{2}$ resulting in difficulties in controlling the disease in the public health sector. Hence, a thorough screening of donated blood using nucleic acid testing for HCV and taking precautions while using medical or health instruments have been considered the best ways to prevent viral transmission. Currently, groundbreaking advancements in anti-HCV therapy have facilitated CHC treatment by lowering the drug adverse effects and improving the sustained virological response rates. ${ }^{3}$ Therefore, extensive screening, early diagnosis, and active treatment of HCV infection using newly developed direct-acting antiviral agents (DAAs) would reduce the prevalence of chronic liver diseases caused by HCV, and will decrease the HCV-related mortality.

In 2017, the World Health Organization (WHO) set the goal of hepatitis elimination and outlined strategies to achieve it by 2030. In this report, hepatitis elimination is defined as a reduction in the incidence of hepatitis and hepatitis-related deaths by

\section{Abbreviations:}

$\mathrm{CHC}$, chronic hepatitis $\mathrm{C}$; DAAs, direct-acting antiviral agents; $\mathrm{HCV}$, hepatitis $\mathrm{C}$ virus; WHO, World Health Organization
$90 \%$ and $65 \%$, respectively, compared to the values reported in 2015 (baseline). ${ }^{4}$ The targets for primary interventions to eliminate HCV infection include achieving 100\% blood safety, $0 \%$ unsafe needle injection, as well as $90 \%$ and $80 \%$ diagnosis and treatment of HCV infection, respectively (Table 1), ${ }^{4}$ although one model suggests even higher targets. ${ }^{5}$ To approach these targets, appropriate policies need to be established, led by the public health ministry of each country. Currently, Australia and New Zealand are obtaining a higher score for nationwide HCV policies as they have appropriate HCV-related data, such as an economic estimate of the burden of viral hepatitis using national epidemiological data as well as programs for disease control, including a publicly funded screening program, government-subsidized HCV treatment, and harm-reduction programs. ${ }^{6}$ These countries are considered to be on the right track for HCV elimination. However, Canada is not considered to be on the appropriate track for hepatitis elimination, despite having high scores for HCV policies. ${ }^{6}$ The reasons include insufficient awareness of HCV, difficult-to-reach health care providers, and inadequate linkage-to-care rates. ${ }^{6}$ Therefore, various aspects should be considered for successful elimination of HCV infection.

There are multiple barriers to the early diagnosis of HCV infec-

\section{Corresponding author: Hyung Joon Yim}

Department of Internal Medicine, Korea University Ansan Hospital, 123 Jeokgeum-ro, Danwon-gu, Ansan 15355, Korea

Tel: +82-31-412-6565, Fax: +82-31-412-5582

E-mail: gudwns21@korea.ac.kr

https://orcid.org/0000-0002-6036-2754 
Table 1. Service coverage indicators for core interventions of the Global Health Sector Strategy on viral hepatitis: 2015 baseline and targets

\begin{tabular}{llccc}
\hline \multirow{2}{*}{ Intervention } & \multicolumn{1}{c}{ Indicator } & 2015 (baseline) & \multicolumn{2}{c}{ Targets } \\
\cline { 3 - 5 } & & $97 \%$ & $\mathbf{2 0 2 0}$ & $\mathbf{2 0 3 0}$ \\
\hline Blood safety & Donations screened with quality assurance & $95 \%$ & $100 \%$ \\
Injection safety & Proportion of unsafe injections & $5 \%$ & $0 \%$ & $0 \%$ \\
\hline Harm reduction & Syringes and needles distributed/PWID/year & 27 & 200 & 300 \\
\hline Testing services & HCV-infected diagnosed & $20 \%$ & $30 \%$ & $90 \%$ \\
Treatment & Diagnosed with HCV started on treatment & $7 \% \%^{*}$ & $-^{\dagger}$ & $80 \%^{\ddagger}$
\end{tabular}

Modified from the global hepatitis report. ${ }^{4}$

PWID, person who injects drugs; HCV, hepatitis C virus.

* Less than $20 \%$ of people living with hepatitis B virus infection are eligible for treatment with antiviral therapy available today.

${ }^{\dagger}$ Three million patients treated for hepatitis C virus infection (cumulative targets).

${ }^{\ddagger}$ Of those eligible for treatment.

tion and getting the patients into treatment, as most patients are asymptomatic. To overcome these limitations, screening subpopulations with high incidence rates, such as injection drug users, prison inmates, or specific age groups, has been undertaken and microelimination has been performed in several countries. ${ }^{7.8}$ Despite the intervention, only a limited number of successful eliminations have been reported at the population level so far. Hence, new attempts to enhance detection of HCV infection and improve linkage to care are required.

The study by Huang et al. ${ }^{9}$ published in the current issue demonstrates a practical care cascade model that improves linkage to care. At a tertiary hospital in Taiwan, they applied in-hospital HCV RNA reflex testing using the same blood samples as those used for anti-HCV antibody test and showed a positive result. A positive HCV RNA test result rendered an automatic real-time appointment followed by a late call-back. In addition, they also conducted HCV treatment in hepatology and non-hepatology departments to enhance the treatment uptake rate. The diagnosis and treatment rate of HCV infection between patients with and without the new strategy were then compared. The application of HCV reflex testing strategy significantly improved the HCV RNA testing rate compared to no application of HCV reflex testing strategy ( $100 \%$ vs. $84.8 \%, P<0.001)$, which was more pronounced in the non-hepatology department than in the hepatology department ( $100 \%$ vs. $23.3 \%, P<0.001)$. Furthermore, the inhospital automatic referral system enhanced the treatment rates, which were also more significant at the non-hepatology department compared with the conventional process $(73.9 \%$ vs. $27.8 \%$, $P=0.001)$. The care cascade was suggested to be an efficient model for improving the rates of $\mathrm{CHC}$ diagnosis and treatment uptake, thereby achieving in-hospital HCV microelimination.
$\mathrm{HCV}$ reflex testing was previously implemented in Spain, and it led to an improved diagnosis rate of $\mathrm{CHC}$. ${ }^{10,11}$ In order to be on the right track for HCV elimination, successful strategies must be shared to other countries. The key obstacles of the program may be funding HCV RNA and genotype testing, as patients themselves may not want to receive HCV reflex testing if they are required to pay for it. Therefore, improvement in the awareness on HCV infection and sustainable health policies with financial support would be the prerequisites for the program. Another limitation would be the insufficient experience of a non-hepatologist for CHC treatment. Although currently available DAAs are mostly pan-genotypic agents, a non-hepatologist may not be an optimal physician to treat newly diagnosed CHC patients. The status of underlying chronic liver disease should be evaluated in detail before initiating treatment with DAAs, and complications of advanced liver diseases need to be further managed. Hence, it would be reasonable to refer new patients to a hepatologist for better $\mathrm{CHC}$ management.

In conclusion, to achieve hepatitis C elimination, efforts must be made to establish an early diagnosis and proper treatment of hepatitis C. The model introduced in this issue is considered to play an important role in actual clinical situations.

\section{Conflicts of Interest}

The author has no conflicts to disclose.

\section{REFERENCES}

1. Thomas DL. Global elimination of chronic hepatitis. N Engl J Med 2019;380:2041-2050. 
2. Liang TJ. Current progress in development of hepatitis $C$ virus vaccines. Nat Med 2013;19:869-878.

3. Liang TJ, Ghany MG. Current and future therapies for hepatitis $C$ virus infection. N Engl J Med 2013;368:1907-1917.

4. World Health Organization (WHO). Global hepatitis report. Geneva: WHO, 2017.

5. Heffernan A, Cooke GS, Nayagam S, Thursz M, Hallett TB. Scaling up prevention and treatment towards the elimination of hepatitis $C$ : a global mathematical model. Lancet 2019;393:1319-1329.

6. Palayew A, Razavi H, Hutchinson SJ, Cooke GS, Lazarus JV. Do the most heavily burdened countries have the right policies to eliminate viral hepatitis B and C? Lancet Gastroenterol Hepatol 2020;5:948953.

7. Boerekamps A, Newsum AM, Smit C, Arends JE, Richter C, Reiss P, et al. High treatment uptake in human immunodeficiency virus/hepatitis C virus-coinfected patients after unrestricted access to direct- acting antivirals in the Netherlands. Clin Infect Dis 2018;66:1352 1359.

8. Bartlett SR, Fox P, Cabatingan H, Jaros A, Gorton C, Lewis R, et al. Demonstration of near-elimination of hepatitis $C$ virus among a prison population: the Lotus Glen Correctional Centre hepatitis C treatment project. Clin Infect Dis 2018;67:460-463.

9. Huang $C F$, Wu PF, Yeh ML, Huang Cl, Liang PC, Hsu CT, et al. Scaling up the in-hospital hepatitis $\mathrm{C}$ virus care cascade in Taiwan. Clin Mol Hepatol 2021;27:136-143.

10. Chevaliez S. Strategies for the improvement of HCV testing and diagnosis. Expert Rev Anti Infect Ther 2019;17:341-347.

11. Crespo J, Lázaro P, Blasco AJ, Aguilera A, García-Samaniego J, Eiros JM, et al. Hepatitis C reflex testing in Spain in 2019: a story of success. Enferm Infecc Microbiol Clin. 2020 May 22. doi: 10.1016/ j.eimc. 2020.03.004. 